\title{
A similarity reduction of the Grad-Shafranov equation
}

\author{
Yuri E. Litvinenko \\ Department of Mathematics, University of Waikato, P.O. Box 3105, Hamilton 3240, New Zealand
}

(Received 22 February 2010; accepted 2 June 2010; published online 8 July 2010)

\begin{abstract}
A direct method for finding similarity reductions of partial differential equations is applied to a specific case of the Grad-Shafranov equation. As an illustration of the method, the frequently used Solov'ev equilibrium is derived. The method is employed to obtain a new family of exact analytical solutions, which contain both the classical and nonclassical group-invariant solutions of the GradShafranov equation and thus greatly extends the range of the available analytical solutions. All the group-invariant solutions based on the classical Lie symmetries are shown to be particular cases in the new family of solutions. (C) 2010 American Institute of Physics. [doi:10.1063/1.3456519]
\end{abstract}

The analysis of similarity reductions of partial differential equations plays an important role in many physical applications. Self-similar reductions not only solve problems with specific initial or boundary conditions ${ }^{1}$ but also serve as intermediate asymptotic solutions to a much wider class of problems. $^{2}$

The classical method for finding a similarity reduction of a given partial differential equation is to use the Lie group method to determine a one-parameter group, admitted by the equation, and the corresponding group-invariant solution. ${ }^{3}$ Recently White and Hazeltine ${ }^{4}$ have used this approach to calculate the complete symmetry group admitted by the Grad-Shafranov equation ${ }^{5,6}$ for the poloidal magnetic flux $u(r, z)$,

$$
u_{r r}+u_{z z}-\frac{1}{r} u_{r}+r^{2} F+G=0
$$

in the particular case of constants $F$ and $G$. The equation describes ideal magnetohydrodynamic tokamak equilibria in which both the pressure and the square of the poloidal electric current are linear functions of $u(r, z)$. Here and in what follows, the same notation as in Ref. 4 is adopted, although $\psi(r, z)$ is more often used in literature to denote the poloidal magnetic flux.

White and Hazeltine ${ }^{4}$ used the symmetry group to generate some new solutions from available solutions and derived three new group-invariant solutions to the GradShafranov equation. Not all interesting similarity reductions can be obtained using the standard Lie group method for finding group-invariant solutions. The well-known Solov'ev ${ }^{7}$ equilibrium, for instance, is not among the Lie groupinvariant solutions to Eq. (1). A nonclassical method of group-invariant solutions ${ }^{8,9}$ generalizes the classical Lie method by analyzing group transformations that do not necessarily map a given partial differential equation into itself. The nonclassical method can lead to additional similarity reductions.

A direct method for finding similarity reductions was proposed by Clarkson and Kruskal. ${ }^{10}$ The method is relatively simple to implement because it does not use group theory. The resulting similarity reductions, however, have been shown to be of both classical and nonclassical symmetry types. ${ }^{11}$

The literature on exact solutions to the Grad-Shafranov equation is extensive. Exact solutions in terms of special functions have been derived for the case of a linear inhomogeneous equation, ${ }^{12-15}$ and series expansion solutions to the homogeneous equation have been developed. ${ }^{16}$ Yet interest in new techniques for solving the equation remains strong. Recent examples include modeling tokamaks and field reversal configurations, ${ }^{17}$ developing axially symmetric magnetohydrodynamic models for astrophysical jets, ${ }^{18}$ and constructing counterexamples to Parker's hypothesis in astrophysics. ${ }^{19}$ Hence it appears useful to present some similarity reductions derived with the direct method ${ }^{10}$ and to compare them with the group-invariant solutions obtained with the classical Lie method. ${ }^{4}$ This is the purpose of this brief communication.

The idea of the direct method is to seek a similarity reduction using the very flexible ansatz ${ }^{10}$

$$
u(r, z)=\alpha(r, z)+\beta(r, z) w[x(r, z)] .
$$

Substituting this form into the original equation and demanding that the result be an ordinary differential equation for $w(x)$ leads to a set of constraints that are used to solve for the functions $\alpha(r, z), \beta(r, z), x(r, z)$, and $w[x(r, z)]$. In the case of Eq. (1), the substitution of Eq. (2) leads to

$$
\begin{aligned}
& \beta\left(x_{r}^{2}+x_{z}^{2}\right) w^{\prime \prime}+\left[\left(2 \beta_{r}-\frac{1}{r} \beta\right) x_{r}+2 \beta_{z} x_{z}+\beta\left(x_{r r}+x_{z z}\right)\right] w^{\prime} \\
& +\left(\beta_{r r}+\beta_{z z}-\frac{1}{r} \beta_{r}\right) w \\
& +\left(\alpha_{r r}+\alpha_{z z}-\frac{1}{r} \alpha_{r}+F r^{2}+G\right)=0
\end{aligned}
$$

As an illustration, consider first the case $\alpha=\alpha(r)$, $\beta=\beta(r)$, and $x=x(z)$. Choosing $w(x)=x$ gives

$$
\beta x_{z z}+\left(\beta_{r r}-\frac{1}{r} \beta_{r}\right) x+\left(\alpha_{r r}-\frac{1}{r} \alpha_{r}+F r^{2}+G\right)=0 .
$$

The equation can be satisfied by choosing $x(z)=z^{2} / 2$, in which case $\alpha$ and $\beta$ must satisfy 


$$
\begin{aligned}
& \beta_{r r}-\frac{1}{r} \beta_{r}=0, \\
& \beta+\alpha_{r r}-\frac{1}{r} \alpha_{r}+F r^{2}+G=0 .
\end{aligned}
$$

The last two equations are solved to give

$$
\begin{aligned}
\beta(r)= & \beta_{0}+\beta_{2} r^{2} \\
\alpha(r)= & \alpha_{0}+\left[\alpha_{2}+\frac{1}{4}\left(\beta_{0}+G\right)\right] r^{2}-\frac{1}{8}\left(\beta_{2}+F\right) r^{4} \\
& -\frac{1}{2}\left(\beta_{0}+G\right) r^{2} \ln r .
\end{aligned}
$$

A particular choice of the remaining integration constants $\alpha_{i}$ and $\beta_{i}$ leads to the well-known Solov'ev ${ }^{7}$ solution,

$$
u(r, z)=\frac{1}{2}\left(r^{2}-G\right) z^{2}-\frac{1}{8}(1+F)\left(r^{2}+\frac{G}{b}\right)^{2} .
$$

Now return to Eq. (3) and choose

$$
x=\frac{z}{r} .
$$

Assume that $\alpha=\alpha(r, z)$ and $\beta=\beta(r)$. Then Eq. (3) is simplified as follows:

$$
\begin{gathered}
\beta\left(1+x^{2}\right) w^{\prime \prime}+\left(3 \beta-2 r \beta_{r}\right) x w^{\prime}+\left(r^{2} \beta_{r r}-r \beta_{r}\right) w \\
+r^{2}\left(\alpha_{r r}+\alpha_{z z}-\frac{1}{r} \alpha_{r}+F r^{2}+G\right)=0 .
\end{gathered}
$$

This equation is satisfied by

$$
\beta(r)=r^{\nu}
$$

for any value of the parameter $\nu$ and by any solutions $w(x)$ and $\alpha(r, z)$ of the following two equations:

$$
\begin{aligned}
& \left(1+x^{2}\right) w^{\prime \prime}+(3-2 \nu) x w^{\prime}+\nu(\nu-2) w=0, \\
& \alpha_{r r}+\alpha_{z z}-\frac{1}{r} \alpha_{r}+F r^{2}+G=0 .
\end{aligned}
$$

Equations (13) and (14) constitute a new similarity reduction of the Grad-Shafranov equation. For example,

$$
w(x)=c_{1}\left(x+\sqrt{1+x^{2}}\right)^{-\sqrt{3} / 2}+c_{2}\left(x+\sqrt{1+x^{2}}\right)^{\sqrt{3} / 2}
$$

is obtained for $\nu=1 / 2$, whereas

$$
w(x)=c_{1}\left[x^{2} \sqrt{1+x^{2}}+3 x \sinh ^{-1}(x)-2 \sqrt{1+x^{2}}\right]+c_{2} x
$$

is obtained for $\nu=3$. Here and in what follows $c_{1}$ and $c_{2}$ are integration constants. The general solution $w(x)$ for an arbitrary $\nu$ can be expressed in terms of the associated Legendre functions,

$$
\begin{aligned}
w(x)= & c_{1}\left(1+x^{2}\right)^{(2 \nu-1) / 4} P_{1 / 2}^{(1-2 \nu) / 2}(i x) \\
& +c_{2}\left(1+x^{2}\right)^{(2 \nu-1) / 4} Q_{1 / 2}^{(1-2 \nu) / 2}(i x) .
\end{aligned}
$$

Clearly $\alpha(r, z)$, defined by Eq. (14), already satisfies the original partial differential Eq. (1). The key point, however, is that new nontrivial solutions to the Grad-Shafranov equation can be generated from any solution to Eq. (14). For example, specifying

$$
\alpha(r, z)=-\frac{1}{8} F r^{4}-\frac{1}{2} G z^{2}
$$

leads to the following solution in the case $\nu=3$ :

$$
\begin{aligned}
u(r, z)= & c_{1}\left[\left(z^{2}-2 r^{2}\right) \sqrt{r^{2}+z^{2}}+3 r^{2} z \sinh ^{-1}\left(\frac{z}{r}\right)\right] \\
& +c_{2} r^{2} z-\frac{1}{8} F r^{4}-\frac{1}{2} G z^{2} .
\end{aligned}
$$

Another simple way to generate new solutions is to use $\alpha=\alpha(r)$, in which case Eq. (14) is solved to give

$$
\alpha(r)=c_{1} r^{2}+c_{2}-\frac{1}{8} F r^{4}-\frac{1}{2} G r^{2} \ln r .
$$

Moreover, if $u(r, z)$ is a solution of the Grad-Shafranov equation, then $u(r, z)+\beta w[x(r, z)]$ is also a solution, which makes it possible to combine solutions with different $\nu$.

The family of solutions above contains both the classical and nonclassical group-invariant solutions of the GradShafranov equation and thus greatly extends the range of the available analytical solutions. It may be of particular importance that all the group-invariant solutions based on the classical Lie symmetries ${ }^{4}$ are in fact particular cases in the new similarity reduction.

Indeed, Eq. (13) with $\nu=0$ is solved to give

$$
w(x)=c_{1} \frac{x}{\sqrt{1+x^{2}}}+c_{2} .
$$

Now taking a particular $\alpha(r)$ from Eq. (20) leads to

$$
u(r, z)=c_{1} \frac{z}{\sqrt{r^{2}+z^{2}}}+c_{2}+\frac{1}{2} G r^{2}(1-\ln r)-\frac{1}{8} F r^{4} .
$$

This is the group-invariant solution $U^{(3)}(r, z)$ presented by White and Hazeltine. ${ }^{4}$

Similarly, Eq. (13) with $\nu=1$ is solved to give

$$
w(x)=c_{1} \sqrt{1+x^{2}}+c_{2} x,
$$

whereas the same equation with $\nu=-1$ is solved to give

$$
w(x)=\frac{c_{1}}{\left(1+x^{2}\right)^{3 / 2}}+c_{2}\left[\frac{x}{1+x^{2}}+\frac{\sinh ^{-1}(x)}{\left(1+x^{2}\right)^{3 / 2}}\right] .
$$

Now taking a linear combination of the two solutions,

$$
w(x)=c_{1} \sqrt{1+x^{2}}+\frac{c_{2}}{\left(1+x^{2}\right)^{3 / 2}},
$$

and using a particular $\alpha(r)$ from Eq. (20) leads to

$$
\begin{aligned}
u(r, z)= & c_{1} \sqrt{r^{2}+z^{2}}+c_{2} \frac{r^{2}}{\left(r^{2}+z^{2}\right)^{3 / 2}} \\
& +\frac{1}{3} G r^{2}-\frac{1}{8} F r^{4}-\frac{1}{2} G r^{2} \ln r .
\end{aligned}
$$

This is the group-invariant solution $U^{(1)}(r, z)$ presented by White and Hazeltine. ${ }^{4}$

Finally, Eq. (13) with $\nu=2$ is solved to give 


$$
w(x)=c_{1} x \sqrt{1+x^{2}}+c_{1} \sinh ^{-1}(x)+c_{2} .
$$

Now using $\alpha(r, z)$ from Eq. (18) leads to

$$
\begin{aligned}
u(r, z)= & c_{1} z \sqrt{r^{2}+z^{2}}+c_{1} r^{2} \sinh ^{-1}\left(\frac{z}{r}\right) \\
& +c_{2} r^{2}-\frac{1}{8} F r^{4}-\frac{1}{2} G z^{2} .
\end{aligned}
$$

This is the group-invariant solution $U^{(2)}(r, z)$ presented by White and Hazeltine. ${ }^{4}$ Note for clarity that the expressions for $U^{(1)}$ and $U^{(3)}$ in the paper by White and Hazeltine ${ }^{4}$ contain several misprints.

To summarize, all the group-invariant solutions to a linear Grad-Shafranov equation, derived using the classical Lie method $^{4}$ are, in fact, particular cases in a new family of solutions, obtained by a direct method for finding similarity reductions of partial differential equations. As demonstrated above, other exact analytical solutions are available, which evidently correspond to nonclassical symmetries.

The focus of this brief communication is on the cases $F=$ const and $G=$ const analyzed by White and Hazeltine. ${ }^{4}$ It should be pointed out, however, that exact analytical solutions can be obtained by the method of separation of variables when $F(u)$ and $G(u)$ are general linear functions of $u$, say $F=F_{0}+F_{1} u$ and $G=G_{0}+G_{1} u{ }^{13,14}$ The direct method, ${ }^{10}$ unfortunately, does not result in new solutions when either $F_{1} \neq 0$ or $G_{1} \neq 0$, at least for the types of similarity reductions considered in this brief communication. Specifically, using $x=x(z)$ in Eq. (3) leads to a solution that is equivalent to that obtained by the separation of variables, whereas using $x=z / r$ in Eq. (3) does not result in any solutions.

By contrast, the direct method ${ }^{10}$ should be expected to be effective in studies of the nonlinear Grad-Shafranov equation. As a simple illustration, note that the same choice of $x=z / r$, together with $\alpha=0$ and $\beta(r)=r^{\nu}$, leads to a similarity reduction for $u(r, z)=\beta(r) w(x)$ if $F(u)=F_{0} u^{1-4 / \nu}$ and $G(u)=G_{0} u^{1-2 / \nu}$. Specifically, Eq. (11) for $w(x)$ is reduced to

$$
\begin{aligned}
& \left(1+x^{2}\right) w^{\prime \prime}+(3-2 \nu) x w^{\prime}+\nu(\nu-2) w+F_{0} w^{1-4 / \nu} \\
& +G_{0} w^{1-2 / \nu}=0 .
\end{aligned}
$$

While the resulting similarity reduction turns out to correspond to a classical group invariance, ${ }^{20}$ it illustrates the simplicity and potential usefulness of the present method. Its application to the Grad-Shafranov equation with nonlinear profiles of the pressure and squared poloidal current may result in exact solutions that fit realistic experimental configurations.

A useful report by the anonymous referee is gratefully acknowledged. This work was supported by NASA (Grant No. NNX08AG44G) and by a research fellowship from the Alexander von Humboldt Foundation.

${ }^{1}$ N. H. Ibragimov, A Practical Course in Differential Equations and Mathematical Modelling: Classical and New Methods. Nonlinear Mathematical Models. Symmetry and Invariance Principles (World Scientific, Singapore, 2009).

${ }^{2}$ G. I. Barenblatt, Scaling, Self-Similarity, and Intermediate Asymptotics: Dimensional Analysis and Intermediate Asymptotics (Cambridge University Press, Cambridge, 1996).

${ }^{3}$ P. Olver, Applications of Lie Groups to Differential Equations (Springer, New York, 1993).

${ }^{4}$ R. L. White and R. D. Hazeltine, Phys. Plasmas 16, 123101 (2009).

${ }^{5} \mathrm{H}$. Grad and J. Rubin, Proceedings of the Second United Nations International Conference on the Peaceful Uses of Atomic Energy, United Nations, Geneva, 1958, Vol. 31, p. 190.

${ }^{6}$ V. D. Shafranov, Sov. Phys. JETP 6, 545 (1958).

${ }^{7}$ L. S. Solov'ev, Sov. Phys. JETP 26, 400 (1968).

${ }^{8}$ G. W. Bluman and J. D. Cole, J. Math. Mech. 18, 1025 (1969).

${ }^{9}$ P. J. Olver and P. Rosenau, Phys. Lett. A 114, 107 (1986).

${ }^{10}$ P. A. Clarkson and M. D. Kruskal, J. Math. Phys. 30, 2201 (1989).

${ }^{11}$ D. Levi and P. Winternitz, J. Phys. A 22, 2915 (1989).

${ }^{12}$ E. K. Maschke, Plasma Phys. 15, 535 (1973).

${ }^{13}$ P. J. Mc Carthy, Phys. Plasmas 6, 3554 (1999).

${ }^{14}$ C. V. Atanasiu, S. Günter, K. Lackner, and I. G. Miron, Phys. Plasmas 11, 3510 (2004)

${ }^{15}$ L. Guazzotto and J. P. Freidberg, Phys. Plasmas 14, 112508 (2007).

${ }^{16}$ S. B. Zheng, A. J. Wootton, and E. R. Solano, Phys. Plasmas 3, 1176 (1996).

${ }^{17}$ A. J. Cerfon and J. P. Freidberg, Phys. Plasmas 17, 032502 (2010).

${ }^{18}$ O. I. Bogoyavlenskij, Phys. Lett. A 276, 257 (2000).

${ }^{19}$ O. I. Bogoyavlenskij, Phys. Rev. Lett. 84, 1914 (2000).

${ }^{20}$ O. V. Kaptsov, Sov. Phys. JETP 71, 296 (1990). 